\title{
Performance Analysis of Unsupervised Classification Based on Optimization
}

\author{
K.Velusamy \\ Department of Computer Science, KSR College of \\ Arts and Science, Tiruchengodu, \\ Tamilnadu, India
}

\author{
R.Manavalan \\ Department of Computer Science, KSR College of \\ Arts and Science,Tiruchengodu, \\ Tamilnadu, India
}

\begin{abstract}
Unsupervised classification is one of the primary research areas in data mining. Clustering algorithm partitions a data set into several groups based on the similarity. Quick reduct algorithm is used to find a minimal feature subset from the original feature space while retaining a suitably high accuracy in representing the original features. Fuzzy-C-Mean (FCM) clustering algorithm is one of the most popular clustering methods since it is an efficient, straightforward, easy to implement and sensitive to initialization. Since, the weakness is easily trapped in local optima. In this paper proposes hybrid Fuzzy c means with an evolutionary algorithm known as Ant Colony Algorithm (ACO) for clustering problem in order to flee from local optima by utilizing the merits of both algorithms FCM and ACO. The experimental results confirm the efficiency of the proposed method
\end{abstract}

\section{Keywords}

Fuzzy Clustering, Ant Colony Optimization, Fuzzy C Means, Particle Swarm Optimization,

\section{INTRODUCTION}

In machine learning, unsupervised classification refers to the problem of trying to find hidden structure in unlabeled data. There is no error or reward signal to evaluate a potential solution, since the unlabeled instances are given to the learner. This distinguishes unsupervised classification from supervised classification and reinforcement classification. Clustering is an unsupervised [1] method which is introduced by Jar dine and Sibson in 1968 with the aim of grouping sets of objects into classes such that similar objects are placed in the same cluster while dissimilar objects are in separate clusters.

Lotfi Zadeh introduced fuzzy theory, which is incorporate with clustering by researcher in order to assign data objects partially into multiple clusters. In the fuzzy clusters the degree of membership depends on the closeness of the data object to the cluster centers. The fuzzy clustering algorithm is Fuzzy CMeans (FCM) which introduced by Bezdek [2] in 1974 and now it is widely used. Even though it is an [3] effective algorithm, but the random selection in center points makes iterative process falling into the local optimal solution easily. For solving this problem, recently evolutionary algorithms such as Genetic Algorithm (GA), Simulated Annealing (SA), Ant Colony Optimization (ACO) and Particle Swarm Optimization (PSO) have been successfully applied.

The rest of this paper is organized the following manner, section 2 presents introduction about rough set and quickreduct algorithm, section 3 illustrate fuzzy c means (FCM), section 4 give out fuzzy particle swarm optimization(FPSO), section 5 deals about hybrid fuzzy c means and fuzzy particle swarm optimization, section 6 converse about fuzzy ant colony algorithm(FACO), section 7 present proposed method that is hybrid fuzzy c means with fuzzy ant colony optimization, section 8 discuss experimental results and analysis and this work is conclude in section IX.

\section{ROUGHSET THEORY}

Rough sets theory [5] was proposed by Z. Pawlak in 1982. It makes an approximation of sets using a collection of elementary sets. No need external parameters to analyze data and to draw conclusions methodology based on Rough set. It offers many opportunities for developing Knowledge Discovery methods using partition properties and the discernibility matrix. In the feature selection problem the major idea is to identify the dispensable and indispensable features, using the discernibility matrix. The purpose of using Rough sets is to find the Core, that is, the set of all indispensable features.

\subsection{Feature selection based on rough set theory}

The reduction of attributes is achieved by comparing equivalence relations generated by sets of attributes. Attributes are detached so that the reduced set provides the same quality of classification as the original. A reduct [6] is defined as a subset $\mathrm{R}$ of the conditional attribute set $\mathrm{C}$ such that $\gamma_{R}(D)=\gamma_{C}(D)$. A given dataset may have many attribute reduct sets, so the set $\mathrm{R}$ of all reducts is defined as:

$$
\begin{gathered}
R_{\text {all }}=\left\{X \mid X \subseteq C, \gamma_{X}(D)=\gamma_{C}(D)\right. \\
\gamma_{X}-\{a\}(D) \neq \gamma_{X}(D), \forall a \in X
\end{gathered}
$$

The intersection of all the sets in $\mathrm{R}_{\mathrm{all}}$ is called the core, the elements of which are those attributes that cannot be removed without introducing more contradictions to the representation of the dataset. For many tasks, a reduct of minimal cardinality is ideally searched for. That is, an attempt is to be made to locate a single element of the reduct set $R_{\text {min }} \subseteq R_{\text {all }}$. The step by step procedure of QuickReduct is given here under.

$$
R_{\text {min }}=\left\{X\left|X \subseteq R_{\text {all }}, \forall Y \in R_{\text {all }},\right| X|\leq Y|\right\}
$$

The QuickReduct algorithm [7] searches for a minimal subset without exhaustively generating all possible subsets. The search begins with an empty subset; attributes which result in the greatest increase in the rough set dependency value are added iteratively. This process continues until the search produces its maximum possible dependency value for that dataset $\gamma_{C}(D)$. Note that this type of search does not 
guarantee a minimal subset and may only discover a local minimum.

\section{METHODS}

\subsection{Fuzzy C Means}

The Fuzzy C-Means algorithm (FCM) is an iterative algorithm that finds clusters in data and which uses the concept of fuzzy membership, [8] instead of assigning a pixel to a single cluster, each pixel will have different membership values on each cluster. It partitions set of $\mathrm{n}$ objects $O=\left\{o_{1}, o_{2}, \ldots . . o_{n}\right\}$ in $\mathrm{R}^{\mathrm{d}}$ dimensional [14] space into c $(1<\mathrm{c}<$

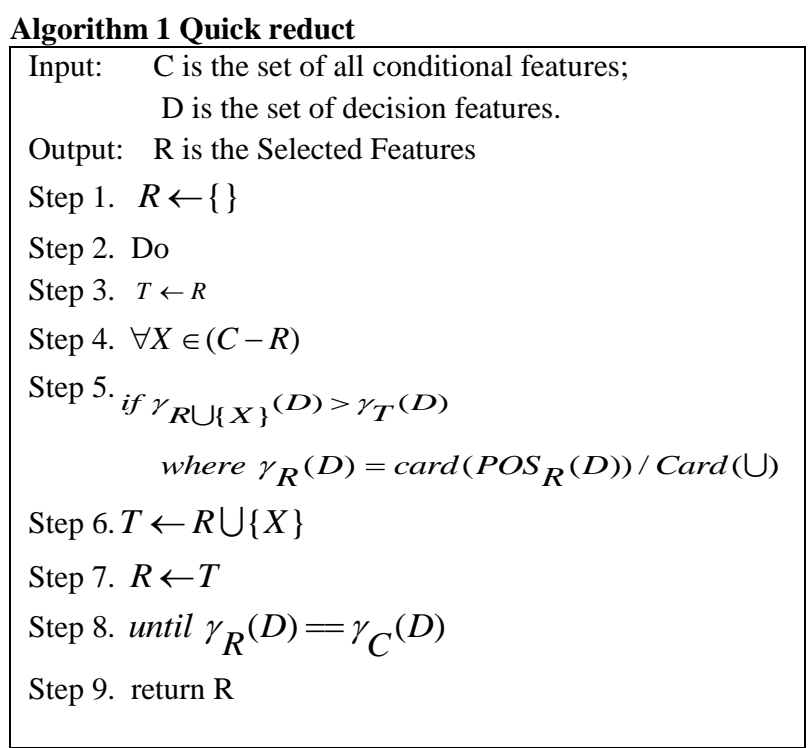

n) fuzzy clusters with $Z=\left\{z_{1}, z_{2}, \ldots ., z_{n}\right\}$ cluster centers. The fuzzy clustering of objects is described by a fuzzy matrix $\mu$ with $\mathrm{n}$ rows and c columns in which $\mathrm{n}$ is the number of data objects and $\mathrm{c}$ is the number of clusters. $\mu_{i j}$, the element in the $i^{\text {th }}$ row and $j^{\text {th }}$ column in $\mu$, point out the degree of association or membership function of the $i^{\text {th }}$ object with the $j^{\text {th }}$ cluster. The characters of $\mu$ are as follows:

$$
\begin{aligned}
& \forall_{i}[0,1] \quad \forall_{i}=1,2 \ldots \mathrm{n} \forall_{j}=1,2 \ldots \mathrm{c} \\
& \sum_{j=1}^{c} \mu_{i j}=1 \quad \forall_{i}=1,2 \ldots \mathrm{n} \\
& o<\sum_{j=1}^{c} \mu_{i j}<n \quad \forall_{j}=1,2 \ldots \mathrm{c}
\end{aligned}
$$

The objective function of FCM algorithm is to minimize the

$$
\begin{array}{r}
\text { Eq. (4): } \quad J_{m}=\sum_{j=1}^{c} \sum_{i=1}^{n} \mu_{i j} d_{i j} \\
\text { where } \quad d_{i j}=\left|o_{i}-z_{j}\right|
\end{array}
$$

From the above equations, $m(m>1)$ is a scalar termed the weighting exponent and controls the fuzziness of the resulting clusters and $d_{i j}$ is the Euclidian distance from object
$Z=\left\{z_{1}, z_{2}, \ldots, z_{n}\right\} \quad$ to the cluster center $z_{j}$. The $z_{j}$, centroids of the $j_{\text {th }}$ cluster, is obtained using below equation

$$
z_{j} \frac{\sum_{i=1}^{n} \mu_{i j}^{m} o_{i}}{\sum_{i=1}^{n} \mu_{i j}^{m}}
$$

There are two stopping rules can be used. First is, if the previous iteration of centroid values and current iteration of centroid values are same, terminate the algorithm, then otherwise go to step 2. And second is the maximum iteration is reached.

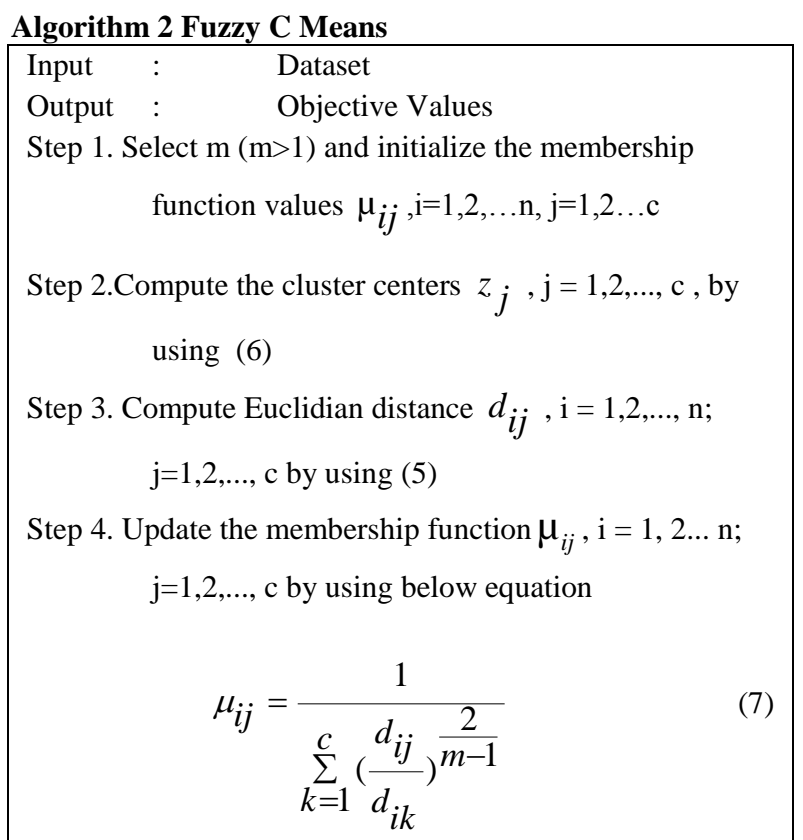

Step 5: If not converged, go to step 2.

\subsection{Particle Swarm Optimization}

Kennedy and Eberhart, in 1995, introduced Particle swarm optimization (PSO) [8] which is population based stochastic optimization technique inspired by bird flocking and fish schooling originally designed and introduced. The PSO starts with a population of particles whose positions represent the potential solutions for the studied problem, and velocities are randomly initialized in the search space. It searches optimal personal best position and global best position in each iteration. The personal best position, pbest, is the best position the particle has visited and gbest is the best position the swarm has visited since the first time step. A particle's velocity and position are updated by using (8).

$$
\begin{gathered}
V(t+1)=w \cdot V(t) \cdot c_{1} r_{1}(\operatorname{pbest}(t)-X(t) \\
+c_{2} r_{2}(\operatorname{gbest}(t)-X(t)) \\
X(t+1)=X(t)+V(t+1)
\end{gathered}
$$


$\mathrm{X}$ and $\mathrm{V}$ are position and velocity [15] of particle respectively, $\mathrm{w}$ is inertia weight, $\mathrm{c} 1$ and $\mathrm{c} 2$ are positive constants, called acceleration coefficients which control the influence of pbest and gbest on the search process, $\mathrm{P}$ is the number of particles in the swarm, $\mathrm{r} 1$ and $\mathrm{r} 2$ are random values range between 0 and 1 .

\subsubsection{Fuzzy Particle Swarm Optimization}

A particle swarm optimization with fuzzy set theory is called fuzzy particle swarm optimization (FPSO), which is proposed by Peng [9] et al.,. Using fuzzy relation between variables, FPSO redefines the position and velocity of particles. And it also applied for clustering problem. In this method $\mathrm{X}$ is the position of particle, the fuzzy relation [16] for the set of data objects, $O=\left\{o_{1}, o_{2}, \ldots, o_{n}\right\}$ to set of cluster centers, $Z=\left\{z_{1}, z_{2}, \ldots, z_{n}\right\}$, Can be expressed as follows

$$
X=\left(\begin{array}{ccc}
\mu_{11} & \cdots & \mu_{1 c} \\
\vdots & \ddots & \vdots \\
\mu_{n 1} & \cdots & \mu_{n c}
\end{array}\right)
$$

Here, $\mu_{i j}$ is the membership function of the $\mathrm{i}^{\text {th }}$ object with the jth cluster with constraints stated (1) and (2) therefore we can see that the position matrix of each particle is the same as fuzzy matrix $\mu$ in FCM algorithm. Also the velocity of each particle is stated using a matrix with the size $n$ rows and $c$ columns the elements of which are in range between -1 and 1 .

The equations (11) and (12) used for updating the positions and velocities of the particles based on the matrix.

$$
\begin{gathered}
V(t+1)=w \otimes V(t) \oplus c_{1} r_{1} \otimes(\operatorname{pbest}(t) \\
-X(t) \oplus c_{2} r_{2} \otimes(\operatorname{gbest}(t)-X(t)) \\
X(t+1)=X(t) \oplus V(t+1)
\end{gathered}
$$

After updating the position matrix, it may violate the constraints given in (1) and (2) since it is compulsory to normalize the position matrix. First we set all the negative elements in matrix to zero. If all elements in a row of the matrix are zero, they need to be reevaluated using series of random numbers within the interval between 0 and 1 , and then the matrix undergoes the following transformation without violating the following constraints:

$$
\text { Xnormal }=\left(\begin{array}{ccc}
\mu_{11} / \sum_{j=1}^{c} \mu_{1 j} & \cdots & \mu_{1 c} / \sum_{j=1}^{c} \mu_{1 j} \\
\vdots & \ddots & \vdots \\
\mu_{n 1} / \sum_{j=1}^{c} \mu_{n j} & \cdots & \mu_{n c} / \sum_{j=1}^{c} \mu_{n j}
\end{array}\right)
$$

This technique uses the fitness function (14) for evaluating the solutions

\section{Algorithm 3.Fuzzy Particle Swarm Optimization}

\begin{tabular}{lll}
\hline Input & $:$ & Dataset \\
Output & $:$ & Objective Values
\end{tabular}

Step 1. Initialize the parameters including population size $P$, $\mathrm{c} 1, \mathrm{c} 2, \mathrm{w}$ and the maximum iterative count.

Step 2. Create a swarm with $P$ particles (X, pbest, gbest and $\mathrm{V}$ are $\mathrm{n} \times \mathrm{c}$ matrices).

Step 3. Initialize $\mathrm{X}, \mathrm{V}$, pbest for each particle and gbest for the swarm.

Step 4. Calculate the cluster centers for each particle using by (6)

Step 5. Calculate the fitness value of each particle using by (14)

Step 6. Calculate pbest for each particle.

Step 7. Calculate gbest for the swarm.

Step 8. Update the velocity matrix for each particle using by (11)

Step 9. Update the position matrix for each particle using by (12)

Step 10. If terminating condition is not met, go to step 4 .

$$
f(X)=\frac{K}{J m}
$$

Here, $K$ is a constant and $J m$ is the objective function of FCM algorithm (4). The smaller is $J m$, the better is the clustering effect and the higher is the individual fitness $f(X)$. The termination condition in this method is the maximum number of iterations or no improvement in gbest in a number of iterations. The algorithm 3 depicted FPSO for clustering problem. The FCM algorithm is quicker than the FPSO algorithm because it need not as much of function evaluations, but it normally go down into local optima. FCM algorithm incorporated with FPSO algorithm to form a hybrid clustering algorithm called FCM-FPSO which maintains the merits of both FCM and PSO algorithms. FCM-FPSO algorithm [10][3] is applied FCM to the particles in the swarm every number of iterations/generations such that the fitness value of each particle is improved.

\subsection{Hybrid Fuzzy C Means and Fuzzy Particle Swarm Optimization}

The FCM algorithm is quicker than the FPSO algorithm because it need not as much of function evaluations, but it normally go down into local optima. FCM algorithm incorporated with FPSO algorithm to form a hybrid clustering algorithm called FCM-FPSO which maintains the merits of both FCM and PSO algorithms. FCM-FPSO algorithm [10][3] is applied FCM to the particles in the swarm every number of iterations/generations such that the fitness value of each particle is improved. The algorithm 4 illustrate hybrid FCMFPSO.

\subsection{Ant Colony Optimization}

Ant algorithms [11] was proposed by Dorigo in 1992 and his colleagues as a multi-agent approach to difficult combinatorial optimization problems like the traveling salesman problem (TSP) and other applications. Path construction and pheromone updating are two major steps in ant colony optimization. In the path construction, ant chooses its path from node i to node $j$. [17] The distance between path 


\section{Algorithm 4 Hybrid FCM-FPSO}

$\begin{array}{lll}\text { Input } & : & \text { Dataset } \\ \text { Output } & : & \text { Objective Values }\end{array}$

Step 1. Initialize the parameters of FPSO and FCM including population size $\mathrm{P}, \mathrm{c} 1, \mathrm{c} 2, \mathrm{w}$, and $\mathrm{m}$.

Step 2. Create a swarm with $\mathrm{P}$ particles (X, pbest, gbest and $\mathrm{V}$ are $\mathrm{n} \times \mathrm{c}$ matrices).

Step 3. Initialize $\mathrm{X}, \mathrm{V}$, pbest for each particle and gbest for the swarm

Step 4. FPSO algorithm

4.1 Calculate the cluster centers for each particle using by (6)

4.2 Calculate the fitness value of each particle using by (14)

4.3 Calculate pbest for each particle.

4.4 Calculate gbest for the swarm.

4.5 Update the velocity matrix for each particle using by (11)

4.6 Update the position matrix for each particle using by (12)

4.7 If terminating condition is not met, go to step 4

Step 5. FCM algorithm

5.1 Compute the cluster centers $z_{j}, \mathrm{j}=1,2, \ldots, \mathrm{c}$, by using (6)

5.2 Compute Euclidian distance $d_{i j}, \mathrm{i}=1,2, \ldots, \mathrm{n}$; $j=1,2, \ldots$, c by using $(5)$

5.3 Update the membership function $\mu_{i j}, i=1,2 . n$ $\mathrm{j}=1,2, \ldots, \mathrm{c}$ by using $(7)$

5.4 Calculate pbest for each particle.

5.5 Calculate gbest for the swarm.

5.6. If FCM terminating condition is not met, go to step 5.

Step 6. If FCM-FPSO terminating condition is not met, go to Step 4

$\mathrm{i}$ and $\mathrm{j}$ is called Euclidean distance which is denoted by $d_{i j}$ and data vector $X_{i}$ is categorized by node i function $p h_{i j}$ represent the pheromone concentration on path from $I$ to $j$ at time of $\mathrm{t}$.

$$
p_{i j}=\left\{\begin{array}{l}
\sum_{j \in s} \frac{p h_{i j}^{\alpha}(t) \cdot \eta_{i j}^{\beta}(t)}{\sum_{o}^{\alpha}(t) \cdot \eta_{i j}^{\beta}(t)} j \in s \\
\eta_{i j}(t)=1 / d_{i j}
\end{array}\right.
$$

The set of all possible paths is represented by $S=\{$ $\left.X_{s} \mid d_{s j} \leq r, \mathrm{~S}=1,2 \ldots \mathrm{N}\right\}$ and $\alpha, \beta$ are two parameters that control the relative weight of the pheromone concentration and the heuristic value. If $\alpha=0$ then the closest path is likely to be selected, which is corresponds to a classical stochastic greedy algorithm. In contrary $\beta$ is zero then only pheromone amplification is at work, this will lead to the rapid emergence of a stagnation situation with the corresponding generation of tours, which are strongly sub-optimal. A trade-off between heuristic value and pheromone concentration come into sight to be compulsory. In the pheromone update step, pheromone concentration on every path is updated according to the following equation,

$$
p h_{i j}(t+1)=\rho p h_{i j}(t)+\Delta p h_{i j}
$$

here, $\rho$ represent the evaporating degree of pheromone concentration with the elapse of time $\Delta p h_{i j}$ is the increase of pheromone concentration with on path(i,j) after one iteration. The value of pheromone evaporation lies between 0 and 1 .

$$
\Delta p h_{i j}=\sum_{k=1}^{n} p h_{i j}^{k}
$$

here, $\Delta p h_{i j}$ is the pheromone concentration left in path (i, j) by the $\mathrm{k}^{\text {th }}$ ant. $\mathrm{k}$ is number of ant.

\subsubsection{Fuzzy Ant Colony Optimization}

The hybridization of ant colony optimization with fuzzy set theory is called fuzzy ant colony optimization proposed. The pheromone is redefined as a fuzzy relationship between variables. Let data vector $X_{i}(\mathrm{i}=1 \ldots \mathrm{n})$ and set of data object, $o=\left\{o_{1}, o_{2}, \ldots, o_{n}\right\}$, to set of cluster $Z=\left\{z_{1}, z_{2}, \ldots ., z_{n}\right\}$. In which $\mu_{i j}$ is the membership function of the $\mathrm{i}^{\text {th }}$ object with $\mathrm{j}^{\text {th }}$ cluster with constraints stated in Eq. (1) and (2) Fuzzy value can express as (3) and Euclidean distance between data item and centroid values that calculated with weight value using equation (20).

$$
P h=\left(\begin{array}{ccc}
\mu_{11} & \cdots & \mu_{1 c} \\
\vdots & \ddots & \vdots \\
\mu_{n 1} & \cdots & \mu_{n c}
\end{array}\right)
$$

$$
d_{i j}=\sqrt{\sum_{k=1}^{m} w_{k}\left(x_{i}-c_{j}\right)^{2}}
$$

where $w_{k}$ is weight factor for each ant, $x_{i}$ is data item, $c_{j}$ is denotes centroid value, $\mathrm{m}$ is the dimension of vector $\mathbf{X}$ and $\mathrm{r}$ the clustering radius. $p h_{i j}(t)$ Represents the pheromone concentration on path $(\mathrm{i}, \mathrm{j})$ at time t. $\eta_{i j}(t)=1 / d_{i j}$ denotes the heuristic value when moving from node $i$ to $j$.

$$
p_{i j}=\left\{\begin{array}{l}
\frac{p h_{i j}^{\alpha}(t) \otimes \eta_{i j}^{\beta}(t)}{\sum_{j \in s} p h_{i j}^{\alpha}(t) \otimes \eta_{i j}^{\beta}(t)} j \in s \\
{ }_{o} \text { otherwise }
\end{array}\right.
$$




\section{Algorithm 5.Fuzzy Ant Colony Optimization}

Step 1. Initialize the parameters of $\beta, \alpha, \mathrm{r}, \mathrm{m}, \mu_{i j}$ and $\mathrm{t}$.

Step 2. Initialize the pheromone concentration and with number of ant.

Step 3. Initialize the clustering center $Z_{j}$ by using (6)

Step 4. For each ant calculates the distance $d_{i j}$ by using (5)

Step 5. If $d_{i j}=0$, probability value is 1 , then go to Step 2 .

Otherwise if $d_{i j}<r$ then calculate heuristic

information by using (17)

Step 6. Find the clustering probability by using (20)

Step 7. Refresh pheromone concentration using (21)

Step 8. Refresh the clustering center using (22)

Step 9. If terminating condition is not met, go to step 4 . Otherwise, end.

$$
p h_{i j}(t+1)=\rho p h_{i j}(t) \oplus \Delta p h_{i j}
$$

If all elements in a row of the matrix are zero, they need to be reevaluated using series of random numbers within the interval between 0 and 1 . Then the matrix undergoes the transformation without violating the constraints as (13)

\subsection{Hybrid Fuzzy C Means and Fuzzy Ant Colony Optimization}

ACO is the well-known swarm intelligent algorithm for solving combinatorial optimization problem and discrete optimization problem. FCM algorithm integrated with FACO algorithm to form a hybrid [13] [18] clustering algorithm called FCM-FACO which keeps the merits of both FCM and ACO algorithms. The procedure is shown in algorithm 6.

\section{Experimental Analysis}

\subsection{Parameter Setting}

FCM, FPSO, FACO, FCM-FPSO, FCM-FACO algorithms are obtained result and performance based on their parameter are selected. In FPSO, $\mathrm{c}_{1}, \mathrm{c}_{2}$ are select the value of 2.0, population is 10 , and weight values are the minimum of weight value is 0.1 and maximum of value is 0.9 . In FACO, $\alpha$, $\beta$ are two parameters that control the relative weight of the pheromone concentration which value is $\alpha=1, \beta=1, \mathrm{r}=0.5, \rho$ $=0.75$ and all algorithms are used $\mathrm{m}$ is 2 .

\subsection{Experimental Analysis}

The algorithms discussed in the previous section have been implemented using MATLAB. For evaluating the all methods are used well known real world dataset is iris have been considered:

- Fisher's iris dataset, which consisting of three different species of iris flower. For each species, 50 samples with four features were collected.

- Wine, which consists of 178 objects and 3 different type classes, each type has 13 features.

Table I is performance of feature selection using quickreduct based on rough set theory and it is select relevant feature from the original feature, Iris dataset has four features and it has selected three features only.

The experimental result of over 100 independent for FCM and 10 independent runs for FPSO, FACO, FCM-FPSO and FCMFACO are summarized in TABLE II based on objective value.

Performance of all cases are illustrate in TABLE II, the hybrid

\section{Algorithm 6.hybrid FCM-FACO}

Step 1. Initialize the parameters of $\beta, \alpha, \mathrm{r}, \mathrm{m}, \mu_{i j}$ and $\mathrm{t}$.

Step 2. Initialize the pheromone concentration and with number of ant.

Step 3. Initialize the clustering center $Z_{j}$ by using (6)

Step 4. FACO algorithm

4.1 for each ant calculates the distance $d_{i j}$ by using (5)

4.2 If $d_{i j}=0$, probability value is 1 , then go to

Step 2.

Otherwise if $d_{i j}<r$ then calculate heuristic

information by using (17)

4.3 Find the clustering probability by using (20)

4.4 Refresh pheromone concentration using (21)

4.5 Refresh the clustering center using (22)

4.6 If terminating condition is not met, go to step

4. Otherwise, end.

Step 5. FCM algorithm

5.1 Compute the cluster centers $z_{j}, \mathrm{j}=1,2, \ldots, \mathrm{c}$, by using (6)

5.2 Compute Euclidian distance $d_{i j}, \mathrm{i}=1,2, \ldots, \mathrm{n}$; $\mathrm{j}=1,2, \ldots, \mathrm{c}$ by using (5)

5.3 Update the membership function $\mu_{i j}, \mathrm{i}=1,2 . \mathrm{n}$, $\mathrm{j}=1,2, \ldots, \mathrm{c}$ by using $(7)$

5.4 go to step 5 .

Step 6. If FCM-FACO terminating is false go to step 4.

Table I-Feature Selection using Quickreduct

\begin{tabular}{|c|c|c|}
\hline Datasets & $\begin{array}{c}\text { No of Feature } \\
\text { before Feature } \\
\text { Selection }\end{array}$ & $\begin{array}{c}\text { No of Feature } \\
\text { after Feature } \\
\text { Selection }\end{array}$ \\
\hline IRIS(150,3,4) & 4 & $3(1,2,3)$ \\
\hline
\end{tabular}

Table II-Objective Value for IRIS Dataset

\begin{tabular}{|c|c|c|c|}
\hline Methods & Worst & Average & Best \\
\hline FCM & 64.28 & 62.92 & 61.12 \\
\hline FPSO & 62.33 & 61.16 & 60.76 \\
\hline FACO & 61.45 & 59.98 & 58.19 \\
\hline FCM-FPSO & 59.33 & 57.29 & 56.93 \\
\hline FCM-FACO & $\mathbf{5 5 . 8 3}$ & $\mathbf{5 4 . 2 2}$ & $\mathbf{5 2 . 8 1}$ \\
\hline
\end{tabular}


FCM-FACO obtained the best results other then all methods and iris dataset is escapes from local optima.

The table II is represents objective value of each method and the bold font values are representing best result compare then other methods. So, FCM-FACO is improved method then other methods.

\section{CONCLUSION}

The clustering problem is a most important problem in data mining. There are many algorithm has been proposed for clustering. In this paper present a new hybrid with fuzzy c means and fuzzy ant colony algorithm has been applied successfully for real world datasets. The computational results show that the performance of the proposal algorithm is better than other existing algorithms.

\section{REFERENCES}

[1] Mehdizadeh.E, Sadi-Nezhad.S and TavakkoliMoghaddam.R, Optimization of Fuzzy Clustering Criteria by a Hybrid PSO and Fuzzy C-Means Clustering Algorithm," Iranian Journal Of Fuzzy Systems Vol. 5, No. 3, (2008).

[2] Jeng-Ming Yih, Yuan-Horng Lin, Hsiang-Chuan Liu,"Clustering Analysis Method based on Fuzzy CMeans Algorithm of PSO and PPSO with Application in Image Data," ISBN: 978-960-474-028-4, Proceedings of the 8th WSEAS International Conference on Applied Computer Science (Acs'08).

[3] Hesam Izakian a, Ajith Abraham," Fuzzy C-means and fuzzy swarm for fuzzy clustering problem," 0957-4174 2010 Elsevier Ltd., doi:10.1016/j.eswa.2010.07.112

[4] Selvi.V Dr.R.Umarani, "Comparative Analysis of Ant Colony and Particle Swarm Optimization Technique," International Journal of Computer Applications (0975 8887)Volume 5- No.4, August 2010.

[5] Zhiding $\mathrm{Yu}$, OscarC $\mathrm{Au}$, RuobingZou, WeiyuYu, JingTian, "An adaptive unsupervised approach toward pixel clustering and color image segmentation," 00313203, 2009 Elsevier Ltd, doi:10.1016/j.patcog.2009.11.015

[6] Velayutham.C and Thangavel.K ," Unsupervised Quick Reduct Algorithm Rough Set Theory "Journal of Electronic Science and Technology, Vol. 9, No. 3, September $2011 \mathrm{Hg}$
[7] Zhang.M, Yao J. T, "A Rough Sets Based Approach to Feature Selection, "University of Regina, Saskatchewan.

[8] Qiang Niu, Xinjian Huang,"An Improved Fuzzy Cmeans Clustering Algorithm based on PSO," Journal Of Software, Vol. 6, no. 5, may 2011.

[9] Peng. W, Wang.k, zhou.c, long.l ,"Fuzzy Disctrete Particle Swarm Optimization For Solving Traval Salsemen Person," In Prceedings Of The Fourth International Conference On Computer And Information Technolong, IEEE Cs Press, pp 796-800(2004).

[10] Hesam Izakian a, Ajith Abraham," Fuzzy C-means and fuzzy swarm for fuzzy clustering problem," 0957-4174 2010 Elsevier Ltd., doi:10.1016/j.eswa.2010.07.112

[11] Chandra Mohan.B. Baskaran.R ,"A survey: Ant Colony Optimization based recent research and implementation on several engineering domain," 0957-4174, 2011 Elsevier Ltd.

[12] Yanfang Han_, Pengfei Shi, "An improved ant colony algorithm for fuzzy clustering in image segmentation," $0925-2312$ doi:10.1016/j.neucom.2006.10.022,2006

[13] Zhiding Yu, OscarC Au, RuobingZou, WeiyuYu, JingTian, "An adaptive unsupervised approach toward pixel clustering and color image segmentation," 00313203, 2009 Elsevier Ltd, doi:10.1016/j.patcog.2009.11.015.

[14] Chen Yanyun, Qiu Jianlin, Gu Xiang, Chen Jianping, Ji Dan Chen Li, "Advances in Research of Fuzzy C-Means Clustering Algorithm”, IEEE, 10.1109/NCIS.2011.104.

[15] Szabo.A, de Castro.L.N," The proposal of a fuzzy clustering algorithm based on particle swarm", Nature and Biologically Inspired Computing (NaBIC), Third World Congress on 2011, IEEE, 978-1-4577-1122-0.

[16] Sanya, Hainan China," SVM Combined with FCM and PSO for Fuzzy Clustering, Seventh International Conference on Computational Intelligence and Security" IEEE, 978-0-7695-4584-4.

[17] Chun-Wei Tsai, Kai-Cheng Hu, Ming-Chao Chiang Chu-Sing Yang, "Ant colony optimization with dual pheromone tables for clustering" Fuzzy Systems (FUZZ), 2011 IEEE International Conference',978-14244-7315-1.

[18] Mohamed Jafar Abul Hasan and Sivakumar Ramakrishnan,"A survey: hybrid evolutionary algorithms for cluster analysis", Artificial Intelegent Review, 179-204, DOI: 10.1007/s10462-011-9210-5 